\title{
Ultra-sonografia transvaginal do colo para a predição do parto pré-termo em pacientes sintomáticas com bolsa integra
}

\author{
Transvaginal ultrasound of the cervix for predicting premature delivery in symptomatic patients \\ with intact membranes
}

\begin{abstract}
Livia Teresa Moreira Rios ${ }^{1}$, Marília da Glória Martins ${ }^{2}$, Rosy Ane de Jesus Barros ${ }^{3}$, Giselly Duailibe Jansen ${ }^{4}$,
\end{abstract} Claudio Rodrigues Pires ${ }^{5}$, Rosiane Mattar ${ }^{6}$

\section{Resumo}

Objetivos: avaliar a utilidade do comprimento cervical ultra-sonográfico para a predição do parto em gestantes com contrações uterinas persistentes e bolsa íntegra. Métodos: realizou-se estudo observacional, prospectivo, do tipo coorte no qual foram incluídas 45 gestantes internadas em nossa instituição entre a $22^{\mathrm{a}}$ e a $34^{\mathrm{a}}$ semana. Ultra-sonografia transvaginal do colo foi realizada uma única vez, após completado um ciclo de tocólise endovenosa, com aferição do comprimento, conforme critérios descritos previamente. Os achados ultra-sonográficos não foram utilizados para o diagnóstico ou conduta obstétrica. O desfecho estudado foi a ocorrência de parto pré-termo ( $<37$ semanas). A análise estatística fundamentou-se em método de regressão logística univariada para determinar a significância do comprimento cervical para a predição do parto pré-termo. O nível de significância adotado foi de $5 \%(\mathrm{p}<0,05)$ e o intervalo de confiança foi de $95 \%$ (IC $95 \%$ ). Resultados: a incidência de parto pré-termo foi de 51,1\% (23/45). O comprimento cervical associou-se de forma significativa ao desfecho da gestação $(\mathrm{p}<0,0001)$. Após construção de curva característica operatória do receptor, um comprimento cervical de $20 \mathrm{~mm}$ revelou-se como melhor ponto de corte para a predição do parto pré-termo (sensibilidade: 86,9\%; especificidade: $81,8 \%$; valor preditivo positivo: $83,3 \%$ e valor preditivo negativo: $85,7 \%$ ). A área calculada abaixo da curva característica operatória do receptor foi de 0,91 (IC 95\%: 0.79-0.97; p<0,0001). Conclusões: nas mulheres com contrações uterinas persistentes e bolsa íntegra, em tratamento para trabalho de parto pré-termo, um comprimento cervical inferior a $20 \mathrm{~mm}$ demonstrou maior probabilidade de parto pré-termo. A ultra-sonografia transvaginal pode melhorar a acurácia diagnóstica em gestantes sintomáticas.

PALAVRAS-CHAVE: Gravidez; Trabalho de parto prematuro; Colo do útero/ultrasonografia

\section{Abstract}

Purpose: to assess the role of ultrasonographic cervical length in predicting premature labor in patients presenting persistent uterine contractions and intact membranes. Methods: a prospective observational cohort study was performed in 45 women admitted to our hospital between 22 and 34 weeks of gestation. Transvaginal sonographic evaluation of the cervix was performed once in the women who had completed a course of parenteral tocolysis. The cervical length was obtained according to criteria reported previously. Cervical sonographic findings were not used in diagnosis and management. Outcome variable was the occurrence of preterm delivery ( $<37$ weeks). Statistical analysis consisted of univariate method

Trabalho desenvolvido no Serviço de Obstetrícia e Ginecologia do Hospital Universitário da Universidade Federal do Maranhão - UFMA - São Luís (MA) - Brasil.

1 Médica Ultra-sonografista; Coordenadora da Clínica de Imagem do Serviço de Obstetrícia e Ginecologia do Hospital Universidade Federal do Mara nhão - UFMA - São Luís (MA) - Brasil.

2 Professora Adjunta da Disciplina de Obstetrícia. Departamento de Medicina III; Chefe do Serviço de Obstetrícia e Ginecologia do Hospital Universitário Federal do Maranhão - UFMA - São Luís (MA) - Brasil.

3 Professora do Departamento de Medicina III; Médica do Setor de Medicina Fetal do Serviço de Obstetrícia e Ginecologia do Hospital Universitário da Universidade Federal do Maranhão - UFMA - São Luís (MA) - Brasil.

4 Médica Ultra-sonografista da Clínica de Imagem do Serviço de Obstetrícia e Ginecologia do Hospital Universitário da Universidade Federal do Maranhão - UFMA - São Luís (MA) - Brasil.

5 Professor do Departamento de Obstetrícia da Disciplina de Medicina Fetal da Universidade Federal de São Paulo - UNIFESP - São Paulo (SP) - Brasil.

6 Coordenadora da Pós-Graduação do Departamento de Obstetrícia da Universidade Federal de São Paulo - UNIFESP - São Paulo (SP) - Brasil.

Correspondência: Livia Teresa Moreira Rios

Avenida do Vale, L-10, Q-35, Ed. Costa Rica, Apto. 801 - Jardim Renascença - 65075-820 - São Luís - MA - Telefones (98) 3227-8234/ 3235-9115

- e-mail: 1tlrios@terra.com.br 
with the purpose of determining the significant contribution of cervical length to the prediction of preterm delivery. The adopted significance level was $5 \%(\mathrm{p}<0,05)$ and the confidence interval was $95 \%(95 \% \mathrm{CI})$. Results: the incidence of preterm delivery was $51.11 \%(23 / 45)$. Cervical length was significantly associated with the outcome $(\mathrm{p}<0.0001)$. Receiver operating characteristic curve analysis showed that a cervical length of $20 \mathrm{~mm}$ was the best cutoff in predicting preterm delivery (sensitivity $86.9 \%$; specificity $81.8 \%$; positive predictive value $83.3 \%$; negative predictive value $85.7 \%$ ). The calculated area under the curve was 0.91 (95\% CI: 0.79-0.97; $p<0.0001)$. Conclusions: among women with persistent uterine contractions and intact membranes treated for preterm labor, a cervical length of less than $20 \mathrm{~mm}$ demonstrated a high likelihood of preterm birth. Transvaginal ultrasound may improve the accuracy of diagnosis in symptomatic women.

KEYWORDS: Pregnancy; Obstetric labor, premature; Cervix uteri/ultrasonography

\section{Introdução}

A incidência de recém-nascidos prematuros em diferentes países permanece inalterada há décadas, apesar dos importantes avanços nos cuidados perinatais. Mantém-se entre 8 e 10\%, variando entre as populações em função dos fatores de risco presentes ${ }^{1}$.

Entre 20 e $30 \%$ dos partos pré-termos são eletivos, por indicações maternas, especialmente hemorragias do terceiro trimestre e síndromes hipertensivas, ou agravos fetais. As principais afecções maternas determinantes da prematuridade "espontânea" são o trabalho de parto pré-termo e a ruptura prematura pré-termo de membranas, de elevada prevalência e de difícil prevenção ${ }^{2}$. A primeira responde por aproximadamente metade e a segunda contribui com $30 \%$ dos partos antes de 37 semanas de gravide ${ }^{3}$.

Acredita-se que o mecanismo responsável pela instalação espontânea do trabalho de parto entre a $20^{\mathrm{a}}$ e a $37^{\mathrm{a}}$ semana seja multifatorial. Agindo sinergicamente, esses fatores fazem com que as contrações uterinas improdutivas, presentes na gravidez, tornem-se produtivas, determinando dilatação cervical com conseqüente expulsão fetal ${ }^{4}$.

Na prática obstétrica, o diagnóstico do trabalho de parto pré-termo é reconhecido pela presença de contrações uterinas persistentes e modificações no colo, apagamento e dilatação ${ }^{5}$. A aferição do colo nessa circunstância é subjetiva e, na maioria das vezes, dificultada pela mudança de posição freqüente durante a gravidez; além disso, em muitas mulheres, o colo é de difícil acessibilidade ao exame vaginal $^{6}$. Essas limitações reduzem os efeitos dos cuidados inibidores do trabalho de parto ${ }^{7}$.

Muitos estudos têm demonstrado que mais de $70 \%$ das mulheres com ameaça de parto pré-termo não progridem para trabalho de parto e parto ${ }^{8}$. Ressaltam ainda que o parto pré-termo ocorre em menos de $10 \%$ das mulheres sintomáticas com bolsa integra. Esse quadro encontra justificativa na carência de critérios clínicos confiáveis para o diagnóstico de trabalho de parto pré-termo ${ }^{9}$.

Além do toque vaginal, a ultra-sonografia transvaginal do colo, realizada após esvaziamento vesical, surge como importante método auxiliar, ao possibilitar a geração de imagens objetivas, que permitem revisão e padronização ${ }^{10,11}$.

Através da medida do comprimento do colo via ultra-sonografia vaginal, há possibilidade de confirmação do apagamento cervical antes do toque vaginal, já que a dilatação do orificio interno só é identificada nesse método quando a abertura está consideravelmente avançada ${ }^{12}$.

Nos casos em que a medida ultra-sonográfica do comprimento do colo foi inferior a $30 \mathrm{~mm}$, a evolução para parto ocorreu dentro de três semanas. A sensibilidade na utilização desse limiar foi de $100 \%$, porém a especificidade foi de $71,4 \%{ }^{4,13}$. Outros autores destacaram que um comprimento cervical inferior a $20 \mathrm{~mm}$ é $100 \%$ preditivo para parto pré-termo, apesar da terapia tocolítica. Estudos mais recentes demonstraram a relevância do limiar de $15 \mathrm{~mm}$ para a predição do parto dentro de sete dias em gestantes sintomáticas ${ }^{9,14,15}$.

Embora se reconheça a medida ultra-sonográfica do comprimento cervical como importante variável auxiliar ao diagnóstico do trabalho de parto pré-termo, contribuindo para minimizar possiveis internações desnecessárias ${ }^{4}$, há controvérsias entre os estudos quanto ao ponto de corte a ser adotado para a predição do parto pré-termo espontâneo em gestantes com contrações uterinas persistentes e bolsa integra ${ }^{16,17}$.

Em função dessas considerações, este estudo tem como objetivo medir o comprimento do colo via ultra-sonografia vaginal nas gestantes com contrações uterinas persistentes e bolsa íntegra internadas na Clínica Obstétrica do Serviço de Obstetrícia e Ginecologia do Hospital Universitário da Universidade Federal do Maranhão (UFMA) para tratamento especializado, procurando determinar o melhor ponto de corte para a predição do parto pré-termo nessa população. 


\section{Métodos}

Realizou-se estudo observacional, prospectivo, do tipo coorte com amostra consecutiva na qual foram incluídas todas as gestantes com contrações uterinas persistentes, bolsa integra e gestação única, entre a $22^{\text {a }}$ e a $34^{\mathrm{a}}$ semana, internadas para tratamento especializado na Clínica Obstétrica do Serviço de Obstetrícia e Ginecologia do Hospital Universitário da UFMA entre de novembro de 2004 e dezembro de 2005.

Foram excluidas as gestantes que apresentaram dilatação cervical superior a $4 \mathrm{~cm}$, sangramento vaginal, polidramnia, malformação congênita, cerclagem prévia, sinais de corioamnionite clínica e restrição de crescimento intra-uterino.

As gestantes selecionadas assinaram o termo de consentimento livre e esclarecido e, em seguida, foram submetidas a estudo ultra-sonográfico.

Os obstetras assistentes determinaram a hospitalização e o tratamento medicamentoso, que consistiu em tocólise com terbutalina endovenosa por 72 horas e corticoterapia antenatal com dexametasona $12 \mathrm{mg} /$ dia intramuscular durante 48 horas.

A idade gestacional foi determinada pela história menstrual, análise de ultra-sonografias anteriores disponíveis, preferencialmente pela medida do comprimento cabeça-nádegas no primeiro trimestre, e pela biometria corrente. Se houvesse discrepância superior a dez dias entre a história menstrual e os exames ultra-sonográficos, a idade gestacional ultra-sonográfica era adotada.

As gestantes foram submetidas a estudo ultra-sonográfico, em média 48 (30-76) horas após a internação na enfermaria de gestantes de alto risco, após completado, no mínimo, o primeiro ciclo de tocólise endovenosa, sendo este o marco zero de entrada da gestante na coorte. Um único examinador realizou o exame ultra-sonográfico para aferir biometria, morfologia e vitalidade fetais, além do comprimento do colo.

O estudo ultra-sonográfico do colo foi realizado pela via vaginal, após completo esvaziamento vesical. A gestante foi colocada em posição ginecológica, em decúbito dorsal, com as pernas abduzidas. Depois de recoberto por preservativo estéril não lubrificado, contendo pequena quantidade de gel transmissor em seu reservatório e sobre sua extremidade, o transdutor foi introduzido lenta e cuidadosamente até o fórnice vaginal anterior e mantido no terço distal do canal vaginal, a fim de se obter vista sagital do colo, com mucosa endocervical evidenciada ao longo do canal cervical, tomando-se o cuidado necessário para não exercer pressão sobre o colo, alongando-o indevidamente. Foram identificados os orificios externo e interno, o canal cervical e a mucosa endocervical.

Caso o orificio interno estivesse fechado, a medida linear do comprimento era efetuada desde a área triangular do orificio interno até a incisura em forma de "V" do orificio externo. Quando o orifício interno se encontrava aberto, com mais de $5 \mathrm{~mm}$, o comprimento do colo funcional, definido como distância da porção ocluída residual, era mensurado sem manobra de compressão fúndica ou suprapúbica (Figura 1). Foram realizadas três medidas, na ausência de mudança cervical dinâmica, sendo a média das medidas do comprimento registrada em milimetros. Caso fosse verificada presença de mudança cervical dinâmica durante o exame, três outras medidas eram realizadas após sua cessação, com média anotada. O tempo médio de exame para o estudo ultra-sonográfico cervical foi de sete minutos.

O resultado do exame ultra-sonográfico do colo via vaginal não foi disponibilizado à equipe médica assistente, que obteve apenas informações ultra-sonográficas rotineiras, a respeito de parâmetros biométricos e morfológicos fetais, além das condições do bem-estar fetal.

As gestantes foram observadas até o parto, identificando-se quais delas pariram antes da $37^{\mathrm{a}}$ semana ou a termo, objetivando comparações quanto a variável independente do comprimento cervical.

O tamanho da amostra foi estimado em 40 gestantes, distribuídas igualmente em cada grupo, admitindo-se uma diferença de $10 \mathrm{~mm}$ entre médias para a variável comprimento cervical nos dois grupos: mulheres que pariram antes da $37^{\mathrm{a}}$ semana e mulheres que pariram a termo. O desvio padrão adotado para aferir a dispersão das médias

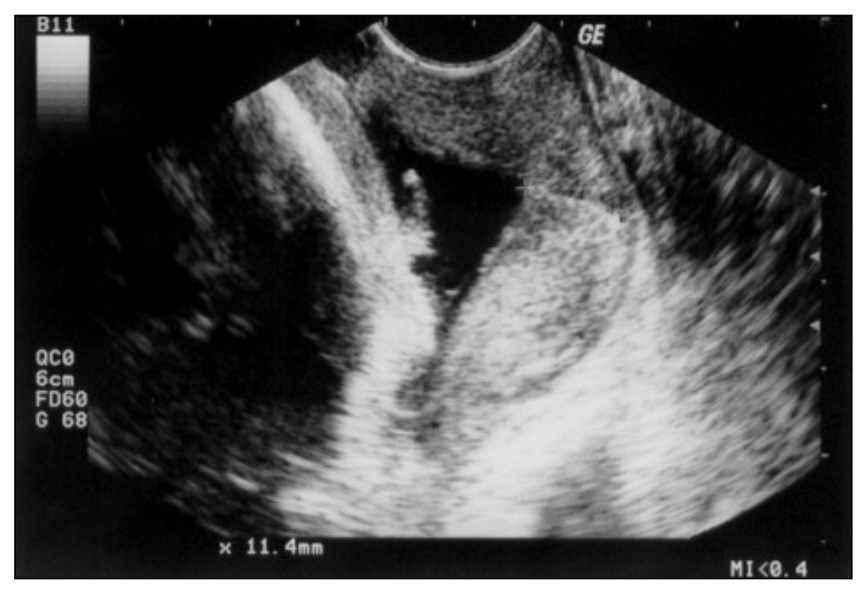

Figura 1 - Medida ultra-sonográfica do comprimento do colo via vaginal. Como o orifício interno se encontra aberto, o comprimento do colo funcional deve ser mensurado, tomando-se o cuidado para não incluir as paredes vaginais na aferição. 
foi de $10 \mathrm{~mm}$. O poder do teste utilizado e o nivel de significância (a) foram fixados em 0,80 e 0,05, respectivamente, para uma análise bicaudal.

Após testar a normalidade das variáveis quantitativas, realizou-se regressão logística univariada, objetivando identificar a significância estatística da associação do comprimento cervical ao parto pré-termo. O nível de significância (a) de $5 \%$ foi adotado para rejeitar a hipótese de independência entre essas variáveis.

Para definir um ponto de corte para o comprimento cervical, observou-se, inicialmente, a mediana. Em seguida, para definir o melhor limiar, foi construída curva de característica operatória do receptor (ROC), após medidas dos índices, sensibilidade e especificidade, adotando-se o ponto de melhor contrabalanço entre eles.

Para avaliar o desempenho do método ultra-sonográfico como teste diagnóstico, foram mensurados sensibilidade, especificidade, valores preditivos e acurácia.

Esta pesquisa foi aprovada pelo Comitê de Ética em Pesquisa do Hospital Universitário da UFMA.

\section{Resultados}

Foram incluídas 52 gestantes com contrações uterinas persistentes e bolsa íntegra internadas na Clínica Obstétrica do Serviço de Obstetrícia e Ginecologia do Hospital Universitário da UFMA. No seguimento, perderam-se sete gestantes. A amostra final deste estudo foi de 45 mulheres.

No momento da entrada na coorte, houve maior percentual de mulheres com idade gestacional entre a $31^{\mathrm{a}}$ e a $34^{\mathrm{a}}$ semana $(26$ mulheres,
$57,8 \%$ ). Entretanto, dentre as pacientes que pariram prematuramente, não houve diferença proporcional entre as que pariram até a $34^{\mathrm{a}}$ semana ou entre a $35^{\mathrm{a}}$ e a $37^{\mathrm{a}}$ semana. Quanto à paridade, predominaram mulheres com um ou mais partos anteriores (26 mulheres, 57,8\%), sem história de parto pré-termo anterior (37 mulheres, 82,2\%).

A maioria das pacientes permaneceu internada por um período inferior a dez dias $(35$ mulheres, $77,8 \%)$. O parto pré-termo ocorreu em 23 mulheres $(51,1 \%)$, sendo $17(37,8 \%)$ no período da hospitalização, em idades gestacionais que variaram entre 25 e 36 semanas. Destas, seis pacientes receberam alta hospitalar após tempo médio de permanência hospitalar de 7 (2-10) dias e, subseqüentemente, foram readmitidas e pariram antes da $37^{\text {a }}$ semana.

Entre os aspectos clínicos de cada grupo segundo o desfecho, parto pré-termo ou parto a termo, não se observou diferença significante. Significância estatística foi verificada na idade por ocasião do parto e no intervalo admissão/parto (Tabela 1).

O comprimento cervical associou-se de forma significativa ao desfecho da gestação. Um comprimento de colo inferior a $20 \mathrm{~mm}$ prevaleceu em $86,9 \%$ das gestantes que pariram prematuramente, enquanto $72,7 \%$ das gestantes que pariram a termo tiveram comprimento cervical igual ou superior a 25 $\mathrm{mm}(\mathrm{p}<0,0001)$.

A Figura 2 mostra a curva ROC do comprimento cervical na amostra estudada. A área calculada abaixo da curva foi de 0,91 (IC 95\%: $0,79-0,97 ; p<0,0001)$. O valor que contempla maior sensibilidade sem aumentar a taxa de falsos-positivos está representado no ombro da curva ROC e corresponde a $20 \mathrm{~mm}$.

Tabela 1 - Características clínicas das gestantes com contrações uterinas persistentes e bolsa íntegra segundo ocorrência de parto pré-termo.

\begin{tabular}{|c|c|c|c|}
\hline & \multicolumn{2}{|c|}{ Parto pré-termo } & \multirow{2}{*}{$p$} \\
\hline & $\operatorname{Sim}(n=23)$ & Não (n=22) & \\
\hline Idade (anos) ${ }^{*}$ & $23(16-31)$ & $24(17-37)$ & 0,61 \\
\hline \multicolumn{4}{|l|}{ Paridade $(n)^{\star *}$} \\
\hline Nulípara & $12(52,2 \%)$ & $7(31,8 \%)$ & \multirow{2}{*}{0,17} \\
\hline Um ou mais partos & $11(47,8 \%)$ & $15(68,2 \%)$ & \\
\hline \multicolumn{4}{|l|}{ Idade gestacional (semanas)* } \\
\hline Na admissão & $30,9( \pm 2,8)$ & $30,5( \pm 3,5)$ & 0,82 \\
\hline No parto & $33( \pm 3,0)$ & $38,9( \pm 1,2)$ & $<0,0001$ \\
\hline Intervalo admissão/parto (dias)* & $14,4( \pm 8,5)$ & $59( \pm 25,8)$ & $<0,0001$ \\
\hline Permanência hospitalar (dias) ${ }^{\star}$ & $8,5( \pm 5,1)$ & $8,9( \pm 4,6)$ & 0,79 \\
\hline
\end{tabular}

*Valor de p obtido pelo teste binomial.

** Valor de $p$ obtido pelo teste do $\chi^{2}$. 


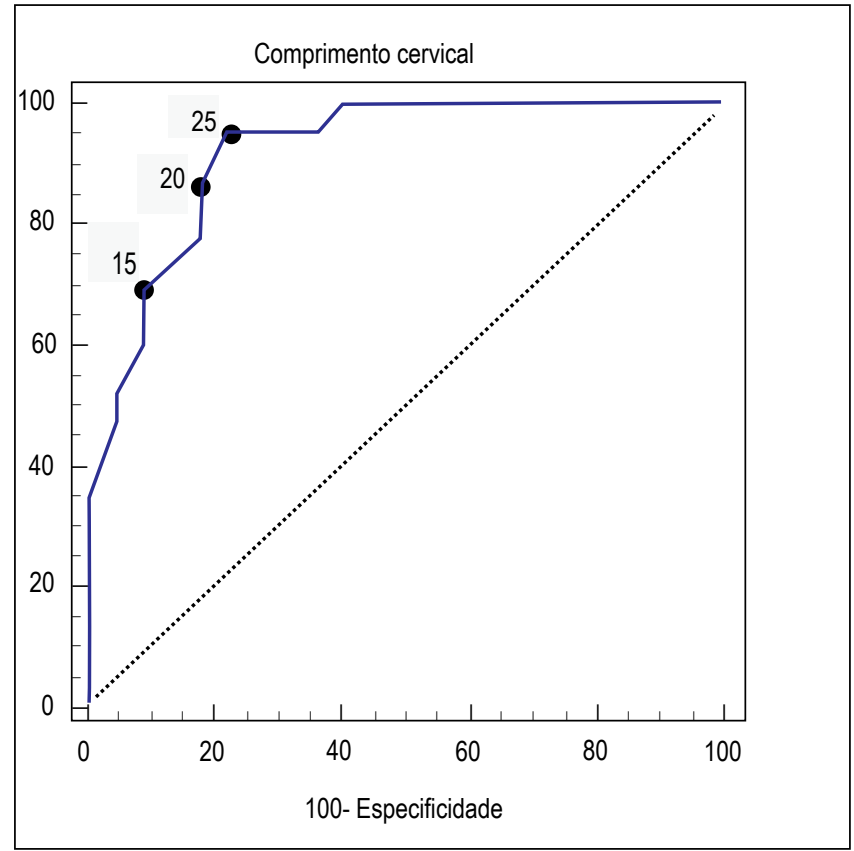

Figura 2 - Curva de característica operatória do receptor do comprimento cervical para a predição do parto pré-termo. Área calculada abaixo da curva: 0,91 (IC 95\%: $0,79-0,97 ; p<0,0001)$.
A Tabela 2 expressa os valores dos índices diagnósticos, sensibilidade e especificidade, para diferentes pontos de corte do comprimento cervical. Observou-se que o melhor contrabalanço ocorreu quando se adotou o limiar de $20 \mathrm{~mm}$, alcançandose sensibilidade de $86,9 \%$ (IC 95\% [66,4-97,1]) e especificidade $81,8 \%$ (IC 95\% [59,7 - 94,7]).

Observou-se que um comprimento cervical inferior a $10 \mathrm{~mm}$ foi $100 \%$ preditivo de parto pré-termo, com valor preditivo negativo de $56,4 \%$, associando-se a uma sensibilidade de $26,1 \%$ e a uma especificidade de $100 \%$. Por outro lado, a predição negativa de $100 \%$ foi alcançada com comprimento cervical igual ou superior a $30 \mathrm{~mm}$, limiar que combinou sensibilidade de $100 \%$ e especificidade de 40,9\% na amostra estudada, com valor preditivo positivo de $63,9 \%$ (Tabela 3 ).

\section{Discussão}

O diagnóstico do trabalho de parto pré-termo freqüentemente torna-se um dilema clínico. Esse quadro encontra justificativa na carência de con-

Tabela 2 - Índices diagnósticos do comprimento cervical de gestantes sintomáticas para a predição do parto pré-termo.

\begin{tabular}{lcc}
\hline Comprimento cervical $(\mathrm{mm})$ & Sensibilidade (IC 95\%) & Especificidade (IC 95\%) \\
\hline$<10$ & $26,1(16,4-57,3)$ & $100,0(84,4-100,0)$ \\
$<15$ & $69,5(47,1-86,7)$ & $90,9(70,8-98,6)$ \\
$<20$ & $86,9(66,4-97,1)$ & $81,8(59,7-94,7)$ \\
$<25$ & $95,6(78,0-99,3)$ & $72,7(54,6-92,1)$ \\
$<30$ & $100,0(85,0-100,0)$ & $40,9(36,4-79,3)$ \\
\hline
\end{tabular}

IC 95\%=intervalo de confiança a $95 \%$.

Tabela 3 - Valores preditivos e acurácia do comprimento cervical de gestantes sintomáticas para a predição do parto pré-termo.

\begin{tabular}{|c|c|c|c|}
\hline Comprimento cervical (mm) & Valor preditivo positivo (\%) & Valor preditivo negativo (\%) & Acurácia (\%) \\
\hline$\geq 30$ & & 100,0 & \multirow{2}{*}{71,1} \\
\hline$<30$ & 63,9 & & \\
\hline$\geq 25$ & & 94,1 & \multirow{2}{*}{84,4} \\
\hline$<25$ & 78,6 & & \\
\hline$\geq 20$ & & 85,7 & \multirow{2}{*}{84,4} \\
\hline$<20$ & 83,3 & & \\
\hline$\geq 15$ & & 74,1 & \multirow{2}{*}{80,0} \\
\hline$<15$ & 88,9 & & \\
\hline$\geq 10$ & & 56,4 & \multirow{2}{*}{62,2} \\
\hline$<10$ & 100,0 & & \\
\hline
\end{tabular}

Rev Bras Ginecol Obstet. 2006; 28(11): 664-70. 
cordância de definições ou de métodos confiáveis para diagnosticá-10 $0^{9,14}$.

A maioria dos estudos realizados em gestantes sintomáticas exclui os casos com avançado trabalho de parto pré-termo, nos quais não há dúvida diagnóstica ou prognóstica. Para tanto, evita-se a inclusão de gestantes nas primeiras 2448 horas da hospitalização. Agindo dessa maneira, restarão os casos duvidosos, cujo comprimento cervical ultra-sonográfico pode ser frutífero, sendo um diferencial na redução da taxa de falso-positivo $^{18-22}$. Com esse procedimento, também adotado em nosso estudo, a realização do exame ultrasonográfico só foi efetivada após, pelo menos, a tocólise endovenosa inicial. Alternativamente, acreditávamos estar minimizando um possivel viés de aferição, já que a medicação tocolitica reduz as contrações uterinas, permitindo a avaliação de um colo menos propenso a modificações determinadas pela ação contrátil miometrial, embora reconheçamos que somente um estudo pareado, aferindo o comprimento do colo antes e depois da tocólise, poderia demonstrar a existência de diferenças estatisticamente significantes.

Em nossa casuística, a incidência de parto pré-termo foi de $51,1 \%$. Essa ocorrência intermediária foi relatada em estudos anteriores ${ }^{12,14,19,23}$, embora outras pesquisas ressaltem que o parto pré-termo ocorre em menos de $10 \%$ das mulheres sintomáticas com bolsa integra ${ }^{9}$, que não é substancialmente diferente da incidência esperada na população assintomática ${ }^{14}$.

Para a definição do melhor ponto de corte para a predição do parto pré-termo nas gestantes com contrações uterinas persistentes e bolsa integra, nas quais o trabalho de parto pré-termo é um diagnóstico plausivel, e a intenção é evitar, na medida do possivel, um resultado falso-positivo, adotamos a estratégia de melhorar a especificidade em detrimento da sensibilidade, sem perder os casos de verdadeiro-positivo. Assumimos, então, o valor de $20 \mathrm{~mm}$ como ponto de corte, mesmo limiar adotado por outros pesquisadores ${ }^{13}$. Entretanto, outros estudos não utilizaram esse critério por priorizarem a sensibilidade e um valor preditivo negativo alto, elevando o ponto de corte ${ }^{18,19,23,24}$.

Verificamos que um comprimento cervical inferior a $20 \mathrm{~mm}$ ocorreu em $86,9 \%$ das gestantes que pariram prematuramente. A adoção desse ponto de corte demonstrou sensibilidade de $86,9 \%$, especificidade de $81,8 \%$, valor preditivo positivo de $83,3 \%$, valor preditivo negativo de $85,7 \%$ e acurácia de $84,4 \%$. Nossos índices diagnósticos e os valores preditivos foram discordantes dos obtidos em pesquisas anteriores, nas quais os valores alcançados foram mais baixos ${ }^{20,25}$ Convém salientar que esses pesquisadores realizaram o estudo ultra-sonográfico ainda na admissão, como teste de rastreamento adjuvante, antes da hospitalização e do início da tocólise endovenosa.

Em nosso trabalho, um comprimento cervical de $30 \mathrm{~mm}$ apresentou-se como o valor que associa sensibilidade de $100 \%$ e especificidade de 40,9\%, com valor preditivo positivo de $63,9 \%$ e valor preditivo negativo de $100 \%$, limiar já sustentado em outros trabalhos ${ }^{12,21,22}$ cujos resultados demonstraram índices diagnósticos e valores preditivos semelhantes. Estudos prévios, adotando o mesmo limiar diagnóstico, encontraram resultados diferentes ${ }^{18,22}$. Possível explicação para essa disparidade reside na utilização de amostra mista, gestações únicas e gestações gemelares.

Houve evidência significativa de associação entre a medida linear da porção cervical ocluída e o desfecho da gestação neste estudo. Portanto, a vigilância cuidadosa de gestantes cujo comprimento do colo se apresenta inferior a $20 \mathrm{~mm}$, após o primeiro ciclo de tocolíticos endovenosos, é prudente. Sem dúvida, o maior benefício do método é a redução de intervenções desnecessárias naquelas com comprimento cervical superior a $30 \mathrm{~mm}$.

Do ponto de vista de pesquisa, seria conveniente determinar se a medida do comprimento cervical deveria ser realizada antes, na admissão, ou depois, após tocólise, ou ainda em outros intervalos temporais seqüenciais. Baseando-se nessa premissa, decidir-se-ia pela hospitalização e administração de tocolíticos e corticosteróides, em função dos custos operacionais e dos riscos materno-fetais, respectivamente.

\section{Agradecimentos}

Agradecemos à professora Dra. Alcione Miranda e a Carlos Serra pelas contribuições na realização deste manuscrito.

\section{Referências}

1. Rozenberg P, Gillet A, Ville Y. Transvaginal sonographic examination of the cervix in asymptomatic pregnant women: review of the literature. Ultrasound Obstet Gynecol. 2002;19(3):302-11.

2. Tucker JM, Goldenberg RL, Davis RO, Copper RL, Winkler CL, Hauth JC. Etiologies of preterm birth in a indigent population: is prevention a logical expectation? Obstet Gynecol. 1991;77(3):343-7.

3. Odibo AO, Ural SH, Macones GA. The prospects for multiple-marker screening for preterm delivery: does transvaginal ultrasound of the cervix have 
a central role? Ultrasound Obstet Gynecol. 2002;19(5):429-35.

4. Iams JD. Cervical ultrasonography. Ultrasound Obstet Gynecol. 1997;10(3):156-60.

5. Gonik B, Creasy RK. Preterm labor: its diagnosis and management. Am J Obstet Gynecol. 1986;154(1):3-8.

6. Michaels WH, Montgomery C, Karo J, Temple J, Acer $\mathrm{J}$, Olson J. Ultrasound differentiation of the competent from the incompetent cervix: prevention of preterm delivery. Am J Obstet Gynecol. 1986;154(3):537-46.

7. Macones GA, Segel SY, Stamilio DM, Morgan MA. Predicting delivery within 48 hours in women treated with parenteral tocolysis. Obstet Gynecol. 1999;93(3):432-6.

8. King JF, Grant A, Keirse MJ, Chalmers I. Betamimetics in preterm labour: an overview of the randomized controlled trials. Br J Obstet Gynaecol. 1988;95(3):211-22.

9. Tsoi E, Akmal S, Rane S, Otigbah C, Nicolaides $\mathrm{KH}$. Ultrasound assessment of cervical length in threatened preterm labor. Ultrasound Obstet Gynecol. 2003;21(6):552-5.

10.Andersen HF, Nugent CE, Wanty SD, Hayashi RH. Prediction of risk for preterm delivery by ultrasonographic measurement of cervical length. Am J Obstet Gynecol. 1990;163(3):859-67.

11.Berghella V, Kuhlman K, Weiner S, Texeira L, Wapner RJ. Cervical funneling: sonographic criteria predictive of preterm delivery. Ultrasound Obstet Gynecol. 1997;10(3):161-6.

12.Murakawa H, Utumi T, Hasegawa I, Tanaka K, Fuzimori R. Evaluation of threatened preterm delivery by transvaginal ultrasonographic measurement of cervical length. Obstet Gynecol. 1993;82(5):829-32.

13.Iams JD, Goldenberg RL, Meis PJ, Mercer BM, Moawad A, Das A, et al. The length of the cervix and the risk of spontaneous premature delivery. N Engl J Med. 1996;334(9):567-72.

14.Tsoi E, Fuchs IB, Rane S, Geerts L, Nicolaides KH. Sonographic measurement of cervical length in threatened preterm labor in singleton pregnancies with intact membranes. Ultrasound Obstet Gynecol. 2005;25(4):353-6.

15. Holst RM, Jacobsson B, Hagberg H, Wennerholm UB. Cervical length in woman in preterm labor with intact membranes: relationship to intraamniotic inflammation/microbial invasion, cervical inflammation and preterm delivery. Ultrasound Obstet Gynecol. 2006;28(6):768-74.

16. Leitich H, Brunbauer M, Kaider A, Egarter C, Husslein P. Cervical length and dilation of the cervical os detected by vaginal ultrasonography as markers for preterm delivery: a systematic review. Am J Obstet Gynecol. 1999;181(6):1465-72.

17. Honest H, Bachmann LM, Coomarasamy A, Gupta JK, Kleijnen J, Khan S. Accuracy of cervical transvaginal sonography in predicting preterm birth: a systematic review. Ultrasound Obstet Gynecol. 2003;22(3): 305-22.

18. Crane JM, Van den Hof M, Armson BA, Liston R. Transvaginal ultrasound in the prediction of preterm delivery: singleton and twin gestations. Obstet Gynecol. 1997;90(3):357-63.

19.Rageth JC, Kernen B, Saurenmann E, Unger C. Premature contractions: possible influence of sonographic meassurement of cervical length on clinical management. Ultrasound Obstet Gynecol. 1997;9(3):183-7.

20.Gomez R, Galasso M, Romero R, Mazor M, Sorokin $\mathrm{Y}$, Gonçalves L, Treadwell M. Ultrasonographic examination of the uterine cervix is better than cervical digital examination as a predictor of the likehood of premature delivery in patients with preterm labor and intact membranes. Am J Obstet Gynecol. 1994;171(4):956-64.

21. Cetin M, Cetin A. The role of transvaginal sonography in predicting recurrent preterm labour in patients with intact membranes. Eur J Obstet Gynecol Reprod Biol. 1997;74(1):7-11.

22.Venditelli F, Mamelle N, Munoz F, Janky E. Transvaginal ultrasonography of the uterine cervix in hospitalized women with preterm labor. Int $\mathrm{J}$ Gynaecol Obstet. 2001;72(2):117-25.

23.Iams JD, Paraskos J, Landon MB, Teteris JN, Johnson FF. Cervical sonography in preterm labor. Obstet Gynecol. 1994;84(1):40-6.

24.Iams JD. Prediction and early detection of preterm labor. Obstet Gynecol. 2003;101(2):402-12.

25.Rizzo G, Capponi A, Arduini D, Lorido C, Romanini C. The value of fetal fibronectin in cervical and vaginal secretions and of ultrasonographic examination of the uterine cervix in predicting premature delivery for patients with preterm labor and intact membranes. Am J Obstet Gynecol. 1996;175(5):1146-51. 